\title{
Estimation of Arrival Time of Coronal Mass Ejections in the Vicinity of the Earth Using SOlar and Heliospheric Observatory and Solar TErrestrial RElations Observatory Observations
}

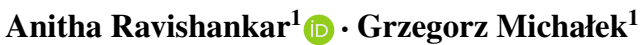

Received: 22 May 2018 / Accepted: 24 May 2019 / Published online: 19 September 2019

(C) The Author(s) 2019

\begin{abstract}
The arrival time of coronal mass ejections (CMEs) in the vicinity of the Earth is one of the most important parameters in determining space weather. We have used a new approach to predicting this parameter. First, in our study, we have introduced a new definition of the speed of ejection. It can be considered as the maximum speed that the CME achieves during the expansion into the interplanetary medium. Additionally, in our research we have used not only observations from the SOlar and Heliospheric Observatory (SOHO) spacecraft but also from Solar TErrestrial RElations Observatory (STEREO) spacecrafts. We focus on halo and partial-halo CMEs during the ascending phase of Solar Cycle 24. During this period the STEREO spacecraft were in quadrature position in relation to the Earth. We demonstrated that these conditions of the STEREO observations can be crucial for an accurate determination of the transit times (TTs) of CMEs to the Earth. In our research we defined a new initial velocity of the CME, the maximum velocity determined from the velocity profiles obtained from a moving linear fit to five consecutive height-time points. This new approach can be important from the point of view of space weather as the new parameter is highly correlated with the final velocity of ICMEs. It allows one to predict the TTs with the same accuracy as previous models. However, what is more important is the fact that the new approach has radically reduced the maximum TT estimation errors to 29 hours. Previous studies determined the TT with a maximum error equal to 50 hours.
\end{abstract}

Keywords Sun: coronal mass ejections (CMEs) - Sun: space weather

\section{Introduction}

Coronal mass ejections (CMEs) play an important role in controlling space weather, which can generate the most intensive geomagnetic disturbances on the Earth (e.g. Gopalswamy

A. Ravishankar anitha@oa.uj.edu.pl

G. Michałek grzegorz.michalek@uj.edu.pl

1 Astronomical Observatory of Jagiellonian University, Krakow, Poland 
et al. 2001b, 2002, Gopalswamy 2002; Srivastava and Venkatakrishnan 2002; Kim et al. 2005; Moon et al. 2005; Manoharan et al. 2004; Manoharan 2006, 2010; Manoharan and Mujiber Rahman 2011; Shanmugaraju et al. 2015). For geomagnetic-storm forecasting it is crucial to predict when a solar disturbance would reach the Earth. This is not an easy task because the rate of expansion of ejections depends on the magnetic force that drives them and the conditions prevailing in the interplanetary medium. In the initial phase, the magnetic force dominates and the ejection is accelerated rapidly. Farther from the Sun, the propelling force weakens and friction begins to dominate. The ejection speed drops gradually approaching the speed of the solar wind. In addition, the ejection velocity can change rapidly as a result of CME-CME interactions. Such collisions mostly occur during a maximum of solar activity.

Initially, models predicting the arrival of interplanetary shocks (IPs) generated by fast CMEs were based on observations of metric Type-II radio bursts (Smart and Shea 1985; Smith and Dryer 1990) but these models were inaccurate (Gopalswamy et al. 1998, 2001a). Gopalswamy et al. (2000a) recognized that the distribution of the speed of interplanetary coronal mass ejections (ICMEs) is much narrower $\left(350-650 \mathrm{~km} \mathrm{~s}^{-1}\right)$ in comparison with the distribution of the speed of CMEs observed near the Sun $\left(150-1050 \mathrm{~km} \mathrm{~s}^{-1}\right)$. This means that CMEs are effectively accelerated as a result of interaction with the solar wind. During expansion, in the interplanetary medium, their speed gradually approaches the speed of the solar wind. Based on these observations, Gopalswamy et al. (2000a) introduced an effective acceleration as the difference between the initial $[u]$ and final $[v]$ speed of an ejection divided by the time $[t]$ taken to reach the Earth. They found a definite linear correlation between the effective acceleration $[a]$ and initial speed of CMEs: $a=1.41-0.0035 u$ ( $a$ and $u$ are in units of $\mathrm{m} \mathrm{s}^{-2}$ and $\mathrm{km} \mathrm{s}^{-1}$, respectively). Gopalswamy et al. (2000b) demonstrated that coronagraphic observations are subject to a major projection effect. To estimate this effect, Gopalswamy et al. (2001b) used archival data from spacecraft in quadrature (Helios 1 and P78-1). This allowed them to improve the relation between $a$ and $u(a=2.193-0.0054 u)$. This relation was used to predict the arrival time of CMEs at 1 AU. It was demonstrated that the highest accuracy was obtained when the acceleration ceased at a distance of $0.75 \mathrm{AU}$. Michałek et al. (2004) further developed this approach to predicting the $1 \mathrm{AU}$ arrival time of halo CMEs. They proposed to determine the effective acceleration only from two groups of CMEs, the fastest and slowest events. These events are assumed to not have acceleration cessation at any place between the Sun and Earth. To minimize the projection effect they also used an innovative method (Michałek, Gopalswamy, and Yashiro 2003) to obtain the real speed of CMEs. This approach allows one to predict the arrival time of halo CMEs with an average error of 8.7 and 11.2 hours for real and projected initial speeds, respectively.

Another precaution to be taken in determining the TTs of the CMEs concerns the moment when a given event reaches the speed of the solar wind (acceleration cessation). However, estimation of the acceleration cessation distance is not an easy task. Few aerodynamic drag models have been developed to solve this problem (Vršnak et al. 2013; Shanmugaraju and Vršnak 2014). These models take into account the difference between speeds of the CMEs and solar wind. Unfortunately, this approach cannot be applied to all CMEs. Using whitelight images and interplanetary scintillations, Manoharan (2006) estimated the TTs for 30 CMEs observed from 1998 to 2004 . He presented important conclusions showing that the TT can be significantly disturbed by CME-CME interactions and changes in solar-wind properties. Recently Syed Ibrahim, Manoharan, and Shanmugaraju (2017) have studied 51 halo and partial-halo CMEs in the ascending phase of Solar Cycle 24 and compared the TT relationship with the initial speed of CMEs in the previous solar cycle, Solar Cycle 23, and the current one: Solar Cycle 24. It has been demonstrated that during the present cycle the 
CMEs have not been significantly affected by the drag force caused by the interplanetary medium.

In our current work, we continue this work using a new approach to a more accurately estimate of the TT of the CME. For this purpose, we use images from SOHO/Large Angle and Spectrometric Coronagraphs (LASCO) and STEREO/Sun Earth Connection Coronal and Heliospheric Investigation (SECCHI) coronagraphs and employed a new technique to determine the speed of ejections. After the prolonged minimum of Solar Cycle 23, the ascending phase of Solar Cycle 24 was observed starting from 2009 with an increase in the number of CMEs. At the same time, the STEREO spacecraft achieved 90 degrees separation relative to Earth, a condition known as quadrature. This location of the spacecraft allows us to give better definition of the parameters of the CMEs, especially of those that are directed towards the Earth (halo CMEs). Additionally, in our studies we introduce a new method for determining the speed of ejections that allows us to estimate the instantaneous speed of CMEs.

This article is organized as follows. The data and method used for the study are described in Section 2. In Section 3, we present results of our study. Finally, the conclusions and discussions are presented in Section 4.

\section{Data and Method}

The main aim of the study is to evaluate the TTs of CMEs to the Earth. For this purpose, observations from the two separate spacecraft, SOHO/LASCO and STEREO/SECCHI, and a new technique to determine the initial speeds of CMEs were employed. Since 1995, CMEs have been routinely recorded by the sensitive LASCO (Brueckner et al. 1995) onboard the SOHO mission. The SOHO/LASCO instruments had already recorded about 30,000 CMEs by December 2016. The basic attributes of CMEs, determined manually from running-difference images, among others, are stored in the SOHO/LASCO catalog (cdaw.gsfc.nasa.gov/CME_list: Yashiro et al. 2004, Gopalswamy et al. 2009). The initial velocity of CMEs obtained by fitting a straight line to the height-time measurements has been the basic parameter used in prediction of the TT. This catalog has been widely used for different scientific studies. Unfortunately, coronagraphic observations of CMEs are subject to projection effects. This makes it practically impossible to determine the true properties of CMEs and therefore makes it more difficult to forecast their geoeffectiveness. This effect mostly affects geoeffective events that originate from the disk center.

Since the launch of STEREO (Kaiser et al. 2008) in 2006 we have a unique opportunity to observe the solar corona from two additional directions. In this study we use these observations to determine velocities of CMEs from additional points of view, since we are concentrating on the events originating in the ascending phase of the Solar Cycle 24. During this period, the STEREO spacecraft were approximately in a quadrature configuration with respect to the Earth. Using quadrature observations with the two STEREO spacecrafts, we can estimate the plane-of-sky speeds which is close to the true radial speed of events ejected from the disk center. This was demonstrated by Bronarska and Michalek (2018). To obtain the STEREO speeds of CMEs we have performed identical manual measurements as in the case of the LASCO observations (Yashiro et al. 2014). The only difference was that for these measurements we used COR2 coronagraphs and the optical telescopes: HI1. To determine the speed of a given CME, we employed only images from the STEREO-A or -B spacecraft, which showed a better quality of observation. This approach allows us to obtain the most accurate height-time data points. As was demonstrated by Michalek, Gopalswamy, 
and Yashiro (2017), the maximal errors in estimation of velocity significantly depend on the quality of CMEs recorded by LASCO coronagraphs. They also demonstrated that a number of height-time points measured for a particular event is the dominant factor in determining the accuracy of CME parameters to the greatest extent. This number is directly dependent on the quality of observations, instrument data gaps, and CME speed. We must also mention that in the case of our considerations it is not important which STEREO spacecraft (A or B) observes a given event because our study is carried out in the period when these twin spacecrafts were in quadrature in relation to the Earth. In addition, these studies are concentrated on halo CMEs that are formed in the central part of the solar disk and are directed towards the Earth. Having the STEREO height-time measurements, we could obtain initial speeds of CMEs from a linear fit recorded by instruments onboard the STEREO spacecrafts. These speeds have been calculated in an identical manner, with the exception of the instruments used, as in the case of those included in the SOHO/LASCO catalog.

It is worth emphasizing here why STEREO and SOHO were used to derive speeds separately. In the current research, we focus on halo CMEs. The STEREO observations in quadrature provide speeds that are very close to spatial (real) velocities, whereas measurements with $\mathrm{SOHO}$ provide speeds that are significantly modified by projection effects. We are interested in how these different speeds can be used to determine the TT of a CME to the Earth.

Until now, empirical models predicting the TTs of the CMEs have employed the initial velocities of ejections obtained from a linear fit to all manually measured height-time data points. Therefore, the determined speeds are in some sense the average velocities of CMEs in the field of view of the respective coronagraphs. It is obvious that the instantaneous velocities of CMEs change with distance from the Sun, since initially their speeds increase when their dynamics is dominated by the Lorentz forces, and they reach their maximum speed when the Lorentz force balances the friction force. From this moment, the CMEs are slowed down until they reach the speed of the solar wind. Therefore, it is evident that the speed determined from the linear fit depends not only on the actual CME speed but also on the number of data points and this significantly depends on the brightness of a given ejection.

In this context, it is worthwhile to estimate the CME speed using a different approach, i.e. to determine the initial speed of CMEs based on their maximal velocities. For this approach, in our current work, we employed a simple technique to determine instantaneous velocities of the CMEs. To obtain these velocities we also used linear fits to height-time points but for now we used a limited number of these points. In our study we considered linear fits using three to eight height-time data points only. Shifting successively, in this way we can obtain the instantaneous speed of the CME. Using such a linear fit, for all of the height-time data points measured for a given $\mathrm{CME}$, we obtained instantaneous profiles of velocities in time or in distance from the Sun.

From the velocity profiles thus obtained, we can easily determine the maximal velocity, as well as the time and the distance when this speed has been achieved. We tested this method employing different numbers of height-time points (from three to eight). The most reasonable results were obtained when we employed five successive height-time points for a linear fit, hence we used this method in our present study. Formally, two neighboring height-time points are enough to calculate the instantaneous speed. Unfortunately manual measurements are subject to unpredictable random errors. These errors result from the subjective nature of manual measurements.

In order to minimize the impact of these errors on the determined instantaneous speed we decided to apply linear fits. This technique allows us to obtain smooth profiles of instantaneous speed. Applying this method we have determined profiles of instantaneous velocities 
of CMEs in the field of view of the LASCO (C2 and C3) and STEREO telescopes. In the case of the STEREO twin spacecraft, we used only observations from the one in which the quality of observation was better. We employed the subscript 5 to denote the use of five successive height-time points for a linear fit to obtain the maximal velocity. These profiles of instantaneous velocities can easily be used to determine the maximal $/ V_{5}$ velocity in the SOHO ( $\left.V_{5-\mathrm{SOHO}}\right)$ and STEREO ( $\left.V_{5-\text { STEREO }}\right)$ telescopes. It should be mentioned that these velocities are the plane-of-sky speeds. However, $V_{5}$-STEREO for halo CMEs is very close to the true radial speed. Having the profiles of instantaneous velocities we estimated the time [ $T_{5-\text { SOHO }}$ and $\left.T_{5-\text { STEREO }}\right]$ and distance $\left[D_{5-\text { SOHO }}\right.$ and $\left.D_{5-\text { STEREO }}\right]$ when the CMEs reached the maximal speeds $\left[V_{5-\mathrm{SOHO}}\right]$ and STEREO $\left[V_{5-\mathrm{STEREO}}\right]$ for the respective coronagraphs.

It is obvious that CMEs, when moving from the Sun to the Earth, are subject to three different phases of propagation: First, close to the Sun, they are subject to rapid initial acceleration [phase $1, S 1]$. At the end of this phase they reach their maximum speeds $\left[V_{5-\mathrm{SOHO}}\right]$ or STEREO [ $V_{5-\text { STEREO }}$. Then, when their dynamics is determined by the drag force (due to interaction with the solar wind), they move with a negative acceleration [phase 2, S2]. After reaching the speed of the solar wind, they move at a constant speed $\left[V_{\mathrm{CONST}}\right.$ : the average speed of the solar wind] until they reach Earth's orbit [phase 3, S3]. Using our definitions we may write

$$
\begin{aligned}
& S 1+S 2+S 3=1 \mathrm{AU}, \\
& S 1=D_{5-\mathrm{SOHO}} / D_{5-\mathrm{STEREO}},
\end{aligned}
$$

where $S 1, S 2$, and $S 3$ are the distances that the CME travels in the next three phases of propagation. In our work, we focus only on the last two phases of propagation. During these phases of propagation a given CME traverses the respective distances: we have

$$
S 2=V_{\mathrm{MAX}} T 2-\frac{a T 2^{2}}{2}
$$

and

$$
S 3=V_{\mathrm{CONST}} T 3,
$$

where $V_{\text {MAX }}$ is the maximal velocity of CMEs achieved in the respective telescopes $V_{5-\mathrm{SOHO}}$ or $V_{5 \text {-STEREO }}, T 2$ is the travel time during the second phase of propagation, $a$ is acceleration during the second phase of propagation, $V_{\mathrm{CONST}}$ is the velocity of propagation during the third phase of propagation, and $T 3$ is the travel time during the third phase of propagation. These equations form the basis of our further considerations, especially those relating to the TTs of CMEs from the Sun to the Earth. In this approach the TT of a CME is $T 2+$ $T 3$ and the distance to travel is $S 1+S 2$. These equations fully describe the kinematics of CMEs in the field of view of the LASCO and SECCHI coronagraphs. The only undefined parameter is the acceleration $[a]$ of CMEs in the second phase of their propagation. Below, we present various methods for its determination. This allows us to test different models used to determine the TTs of CMEs from the Sun to the Earth.

In the article we consider different methods to determine the velocities of the CMEs. These velocities can be correlated with the TTs. Fitting curves to TT-velocity points we built theoretical models that can be used to predict the TT in the future. For individual CMEs, we can determine the error in the estimation of TT as the difference between the TT determined on the basis of the model and the actual observations. Having these errors for a given model and entire populations of considered CMEs, we can determine the average absolute and maximal errors. This means that the maximal error for a given model is the maximal error from the distribution. 


\section{Results}

Our study concentrates on the ascending phase of Solar Cycle 24. During this period we were able to record, at the same time, CMEs observed by STEREO-A and -B spacecraft as they were separated by 90 degrees with respect to Earth. Syed Ibrahim, Manoharan, and Shanmugaraju (2017) compiled a list of halo and partial-halo CMEs during 2009-2013. Among them, they were able to identify the ICME at the Earth's vicinity for 51 events. These events are the basis of our study. Their analyses were limited to the data included in the SOHO/LASCO catalog. Additionally, in our research, for each CME included in their list, we conducted the analysis which was described in the previous section. This means that for each CME we have measured height-time points in the STEREO field of view and the initial ejection velocities of CMEs [ $V_{\mathrm{AVG}}$-STEREO and $V_{5 \text {-STEREO }}$ ] were determined. A few events were too faint in the STEREO images so we were not able to obtain height-time points for them. Having the profiles of instantaneous velocities, we estimated the time and distance when the CMEs reached their maximal speeds. All of these data are shown in Table 1. The near-Sun observational details in the LASCO field of view are given in columns two-seven. The onset date and time of CME ejection are in columns two and three, respectively. The average velocity from a linear fit to all data points (from the SOHO/LASCO catalog), the maximal velocity from linear fit to five successive height-time points, distance, and the time when a given CME reaches the maximal velocity are displayed in columns four - seven, respectively. Next, the TTs of ICME and shocks and final velocity of ICME in the vicinity of Earth received by the in-situ observations made by Advanced Composition Explorer (ACE) instruments are given in columns eight - ten, respectively. These data are from Syed Ibrahim, Manoharan, and Shanmugaraju (2017). The details from observations in the STEREO field of view are shown in columns $11-14$. In the respective columns we find that the average velocity from a linear fit to all data points, the maximal velocity from linear fit to five successive height-time points, distance, and the time when a given CME reaches the maximal velocity. The data shown in the table are the basis for our calculation of the TT of a CME from the Sun to the Earth. The results are presented in the following sections. In the table are included all CMEs (51) considered by Syed Ibrahim, Manoharan, and Shanmugaraju (2017) having recognized magnetic cloud structure at the Earth (having determined the TT for an ICME). For 48 and 39 (including interacting events) of them we were able to obtain the maximal velocities $\left(V_{5-\mathrm{SOHO} / \mathrm{STEREO}}\right)$ in the SOHO/LASCO and STEREO fields of view, respectively.

\subsection{Velocities of CMEs}

In the previous sections, we presented methods for determining the initial speeds of ejections. In this section we present the relationship between these speeds obtained from SOHO and STEREO images. In Figure 1, the successive panels show the relationships between the average and maximal $/ V_{5}$ speeds determined in SOHO images, the average and maximal $/ V_{5}$ speeds determined in the STEREO images, the average speeds determined in SOHO and STEREO images and the maximal $/ V_{5}$ speeds determined in SOHO and STEREO images. Dashed lines are linear fits to data points. Formulas representing linear fits are placed in the lower-right corners of each panel. It can be seen that the average ejection velocities are strongly correlated with their maximal velocities (Panels a and b), regardless of the instrument used for their determination. We can notice that the maximal velocities are much larger (on average $80 \%$ ) than the average velocities in the case of observations from the STEREO spacecraft (Panel b). For SOHO observations the maximal velocities are on average only 


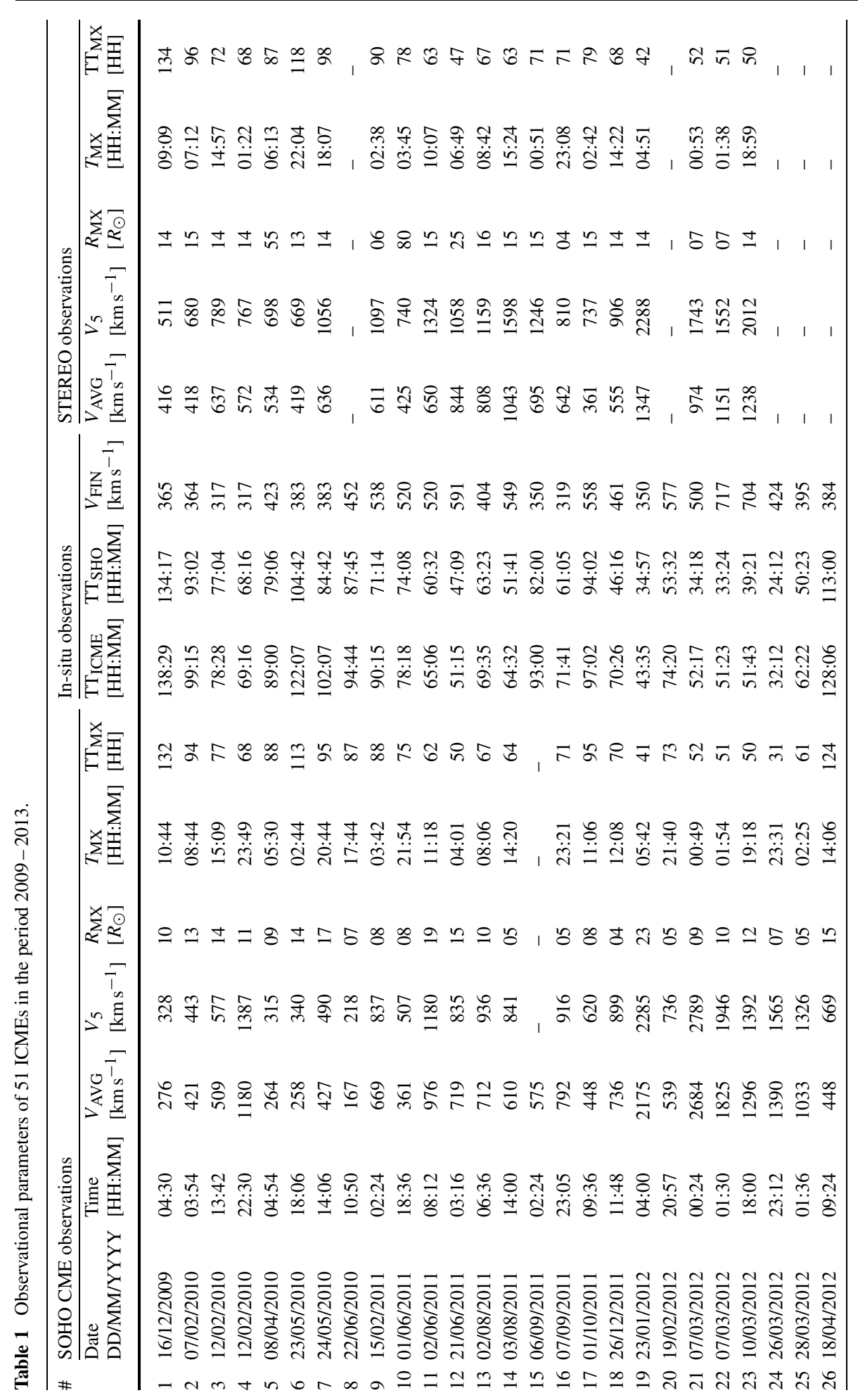




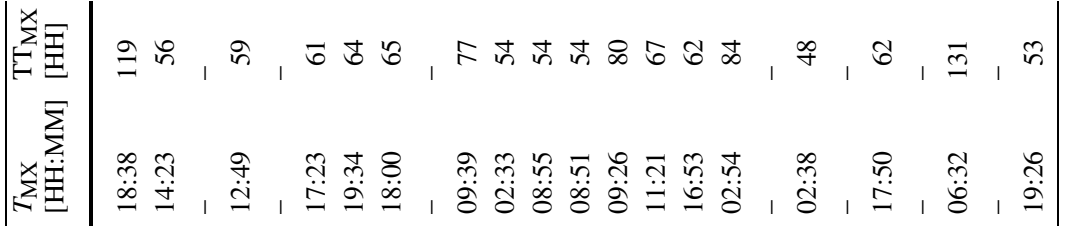

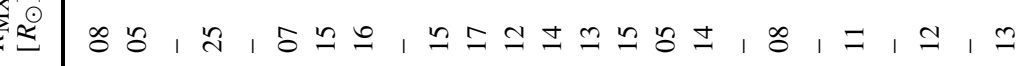
密焉 垔 的

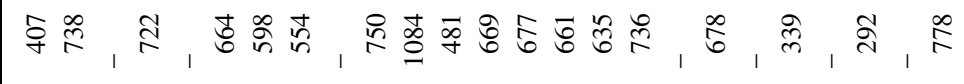

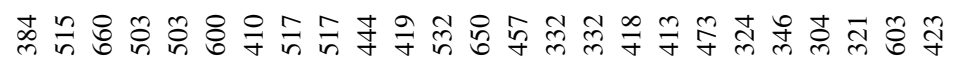
$\Sigma$ \&

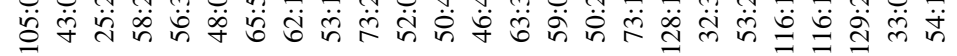
\&

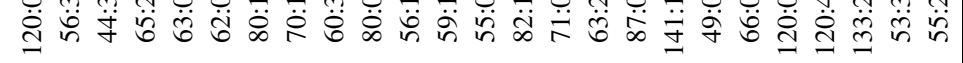

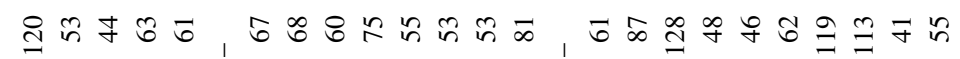

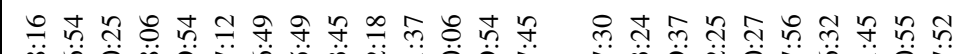

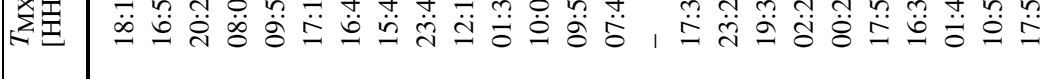

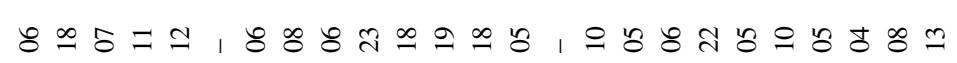
T

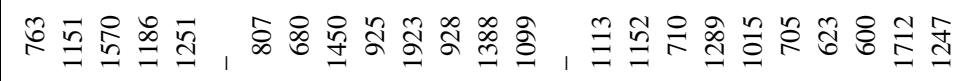

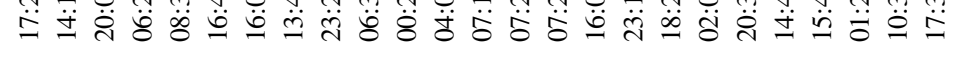

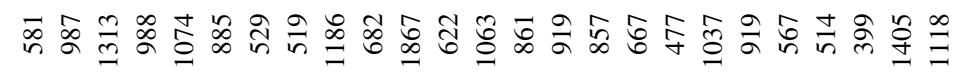

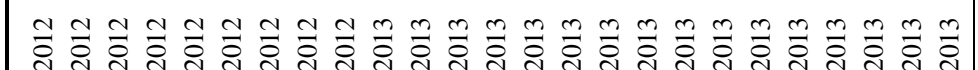

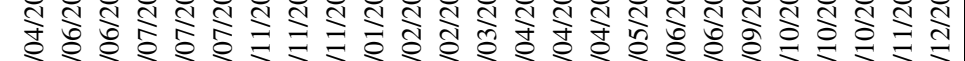

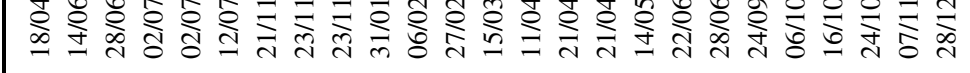
\#

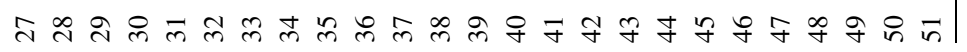



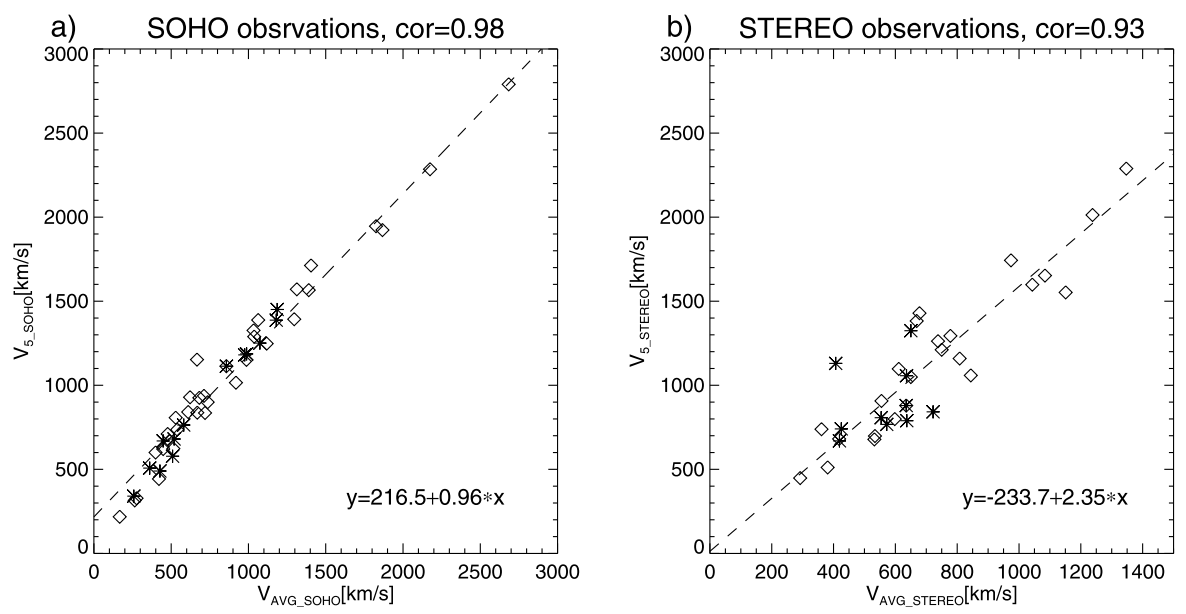

c) SOHO-STEREO obsrvations, cor $=0.76$
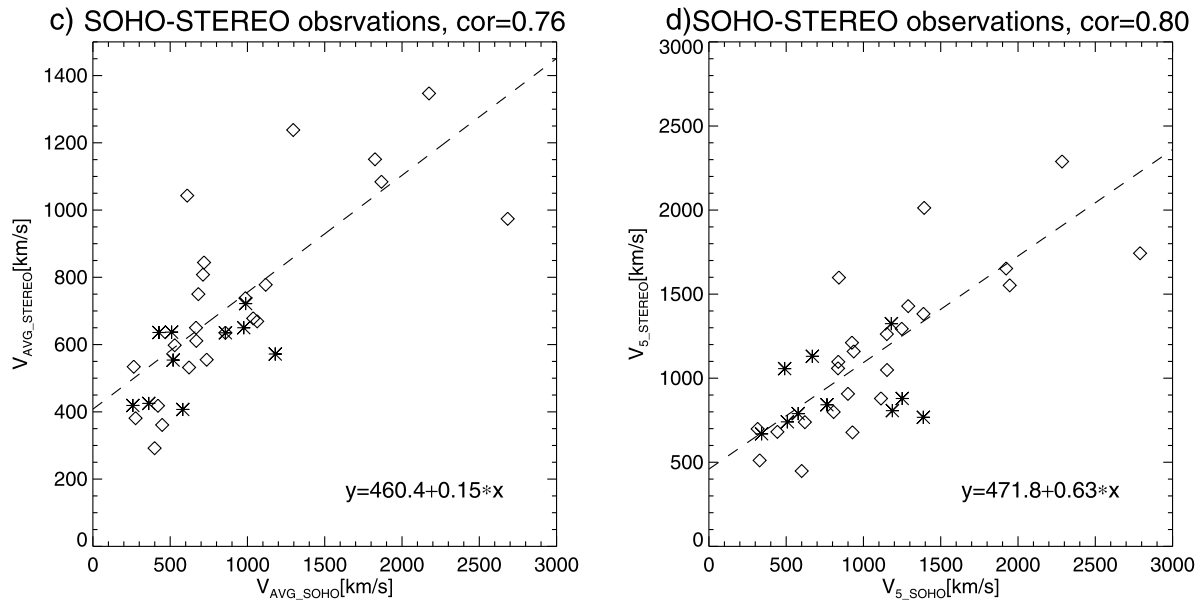

Figure 1 Relationship between (a) the average and maximal $/ V_{5}$ speed determined in SOHO images, (b) the average and maximal $/ V_{5}$ speed determined in the STEREO images, (c) the average speed determined in SOHO and STEREO images, (d) the maximal $/ V_{5}$ speed determined in SOHO and STEREO images. Dashed lines are linear fits to data points. Formulas representing these linear fits are placed in the lower-right corners of each panel. Open diamond symbols are for non-interacting and star symbols are for interacting CME, respectively.

$25 \%$ larger than the average velocities. This results from the fact that the field of view of the STEREO instruments used for determining the velocity profiles (COR2 and HI1) is much larger than the field of view of the LASCO coronagraphs $(\mathrm{C} 2+\mathrm{C} 3)$. It means that the field of view of the STEREO telescopes covers the area where CMEs undergo significant deceleration due to interaction with the solar wind. For this reason, the average velocities of the CMEs determined from the STEREO observations are significantly lower than the other speeds determined in these studies.

Correlations between the speeds for these two instruments are slightly smaller. The correlation coefficients are 0.75 and 0.80 , respectively, for the average and maximal speeds. In this case, the larger dispersion of speeds results from the fact that they are determined from the two different instruments (SOHO and STEREO) that observe the Sun at different 

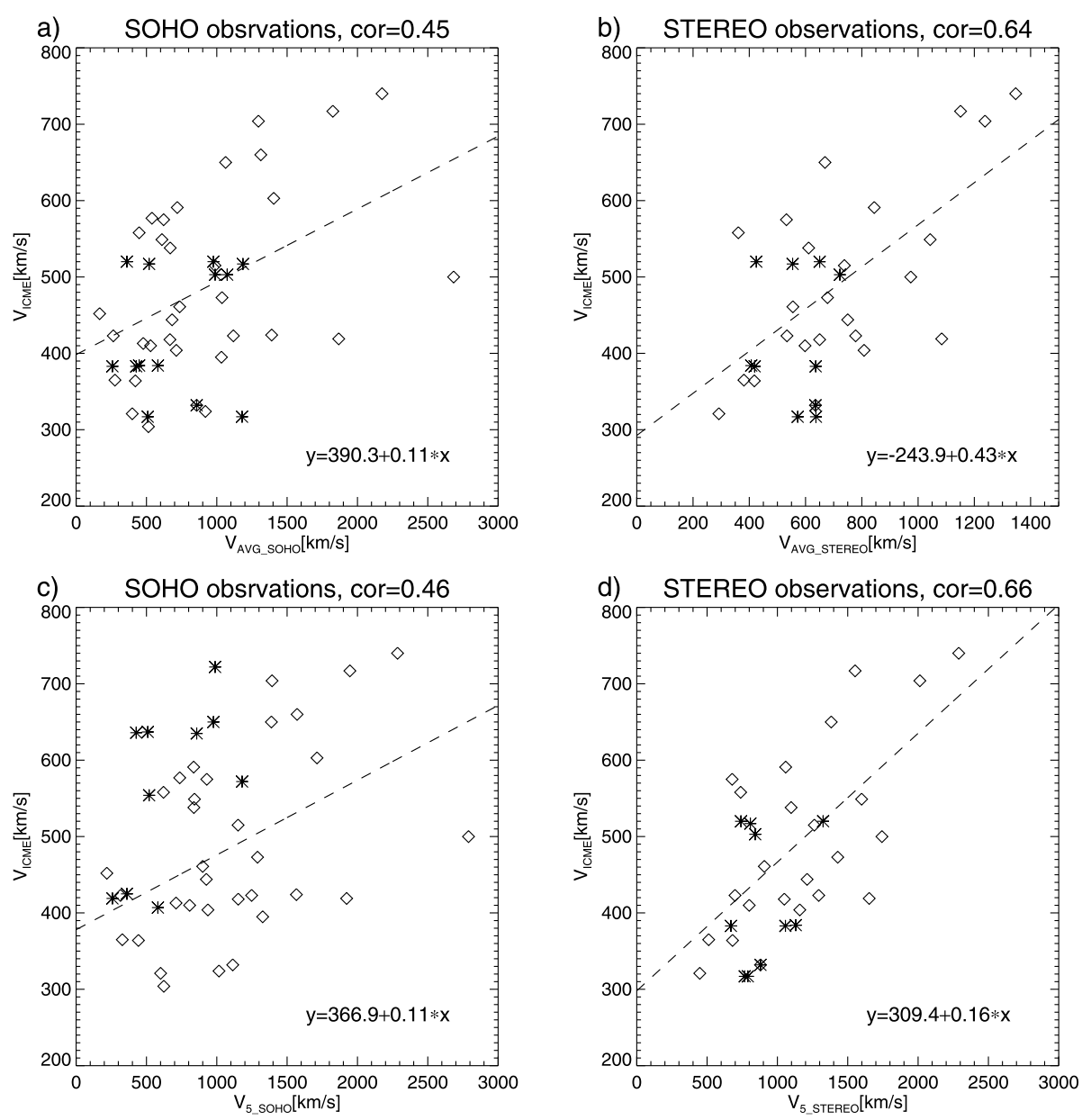

Figure 2 The scatter plots of the average and maximal $/ V_{5}$ speeds determined in SOHO and STEREO images versus ICME speeds determined near the Earth. Dashed lines are linear fits to data points. Formulas presenting these linear fits are placed in the lower-left corner of each panel. Open diamond symbols are for non-interacting and star symbols are for interacting CMEs, respectively.

angles. Depending on the source location on the solar disk and the position of the spacecraft, the determined speeds are subject to different projection effects (Bronarska and Michalek 2018). This effect, among others, is the reason that the determined speeds may be different for each of the telescopes. In our considerations $V_{5-\text { SоHо }}$ are on average smaller (about $50 \mathrm{~km} \mathrm{~s}^{-1}$ ) in comparison with $V_{5-\text { STEREO }}$. This is due to projection effects. Earth-directed $\mathrm{CMEs}$ recorded in SOHO/LASCO coronagraphs are subject to more significant projection effects in comparison with observations by the STEREO spacecraft in a quadrature position.

In Figure 2, the relationships between the initial speeds and speeds of interplanetary coronal mass ejections (ICMEs) obtained from in-situ measurements are shown. In successive panels we have displayed scatter plots of the average velocities and the maximal $/ V_{5}$ velocities determined by SOHO and LASCO telescopes versus ICME speeds recorded near the Earth. 
For the initial speed determined in the SOHO images, the correlation coefficients are less than 0.5. However, in the case of the STEREO spacecraft these correlation coefficients are significantly higher (up to 0.66). It is worth noting that the most significant correlation is between the final speeds of ICMEs and maximal $/ V_{5}$ speeds obtained from the STEREO images (Panel d). From the point of view of space weather this is a new and very important result. The speed of an ICME is one of the most important parameters determining the geoeffectiveness of CMEs. These relations for speeds obtained from the STEREO telescopes allow for a more precise estimation of ICME velocities in the vicinity of the Earth and thus the prediction of their impact on the Earth becomes more accurate.

\subsection{Velocities and Transit Time to the Earth}

Depending on the velocity used, in our current research, we define the TT of a CME in two ways. For the average velocities determined in the STEREO or SOHO images, the TT is the time difference between the CME onset time in LASCO-C2 field of view [ $\left.T_{\mathrm{CME}}\right]$ and the ICME arrival time $\left[T_{\mathrm{ICME}}\right]$ at the Earth's vicinity using in-situ observations [TT = $\left.T_{\mathrm{CME}}-T_{\mathrm{ICME}}\right]$. In the case of maximal velocities obtained in the STEREO or SOHO images, the TT is the difference between the time when the CME reaches its maximal speed [ $\left.T_{\mathrm{MAX}}\right]$ and the ICME arrival time $\left[T_{\mathrm{ICME}}\right]$ in the Earth's vicinity using in-situ observations [TT = $\left.T_{\mathrm{MAX}}-T_{\mathrm{ICME}}\right]$. The TTs for shock generated by ICMEs are obtained in similar ways. The relationships between TTs and CME speeds are shown in the following figures.

As shown in Figure 3, the TTs for CMEs with speeds below $1000 \mathrm{~km} \mathrm{~s}^{-1}$ are in the range 50-140 hours. The TTs for fast events $\left(V>1000 \mathrm{~km} \mathrm{~s}^{-1}\right)$ are in the range $40-$ 60 hours. The third-order polynomial relationship between the observed TT and the speed is indicated by a dashed curve. This fitting can be considered as an empirical model to predict the TT. The difference between the observed and predicted TTs for a given CME speed can be considered as an error by the model. The lower panels show the distribution of errors in determining the TT for this empirical model. The average errors are about $\approx 11-$ 12 hours and the maximum errors are very significant and reach values $\approx 50$ hours. These errors are the maximum of the distribution of errors. Maximum errors were determined as the maximum difference in time between the theoretical model and the observational data. These errors were determined only for non-interacting CMEs.

In Figure 4 we display the same data but for the maximal velocity $/ V_{5}$ obtained from the SOHO images. As shown in the figure, results are similar but the average errors are slightly higher, $\approx 13$ hours. This means that the introduction of the maximal velocity of CME has no effect on a more accurate TT prediction. Figures 3 and 4 and the above discussion refer to SOHO observations.

In Figure 5, results for the average speeds determined in the STEREO images are presented. As shown in the figure, the TT is significantly related to the average speed. The data points are not scattered around the empirical model represented by a third-order polynomial fit. This fit is represented by a dashed curve. This empirical model can be used, with great accuracy, to predict TTs of CMEs. In this case the average errors in the prediction of the TT are only $\approx 9$ and 10 hours for ICMEs and IP shock, respectively. However, a more significant fact is that in this case the maximal errors are much lower than in the previously presented models (Gopalswamy et al. 2001b; Michałek et al. 2004; Manoharan 2006), i.e. 29 and 38 hours for ICME and IP shock, respectively. Similar results were obtained for the maximal $/ V_{5}$ speed in the STEREO images. Results for these considerations are shown in Figure 6. 
a) avg velocity from $\mathrm{SOHO}, \mathrm{ICME}$
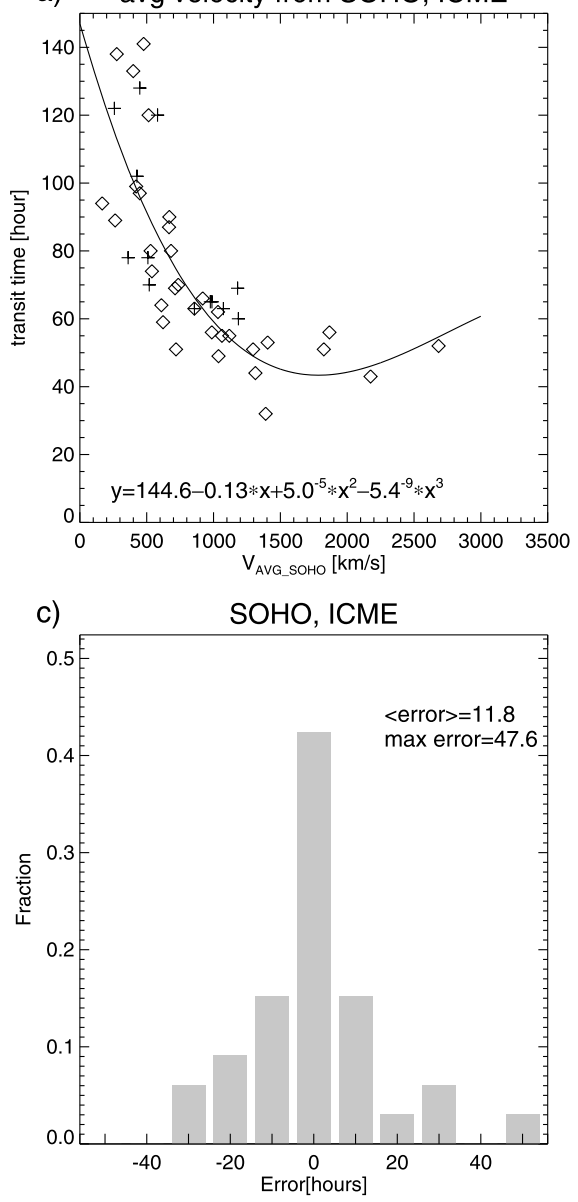

b) avg velocity from SOHO, Shock
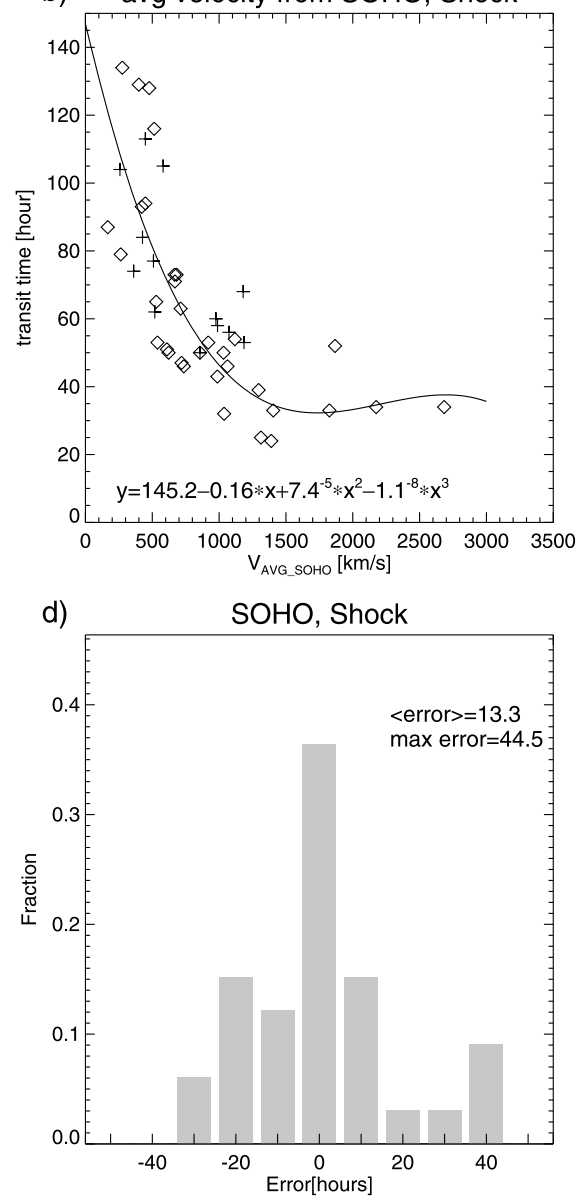

Figure 3 Relationship between the average CME speed obtained in the SOHO images and the observed TT for ICME (Panel a) and IP shock (Panel b). Open diamond symbols are for non-interacting and star symbols are for interacting CMEs, respectively. The third-order polynomial relationship between the observed TT and the speed is indicated by a dashed curve. The distributions of errors between predicted and observed TTs are presented in the bottom panels (Panel $\mathbf{c}$ for ICME and Panel $\mathbf{d}$ for IP shock). The values of the average and maximal errors are presented in the panels.

It is also worth mentioning that in the case of fast CMEs $\left(V>1200 \mathrm{~km} \mathrm{~s}^{-2}\right)$, the empirical model is a better approach to predicting TTs. For these events, the average errors are below five hours and the maximal error does not exceed ten hours. This is illustrated in the figure by two additional dashed curves showing a deviation from the model of \pm ten hours.

\subsection{CME Transit Time Estimation}

Having determined the different initial velocities (maximal or average), we are able to calculate TTs directly using kinematic equations of motion. For this purpose, we assume that once the CME reaches the initial velocity it moves with a uniform negative acceleration. This movement is controlled by drag force due to interaction with the solar wind. Decel- 

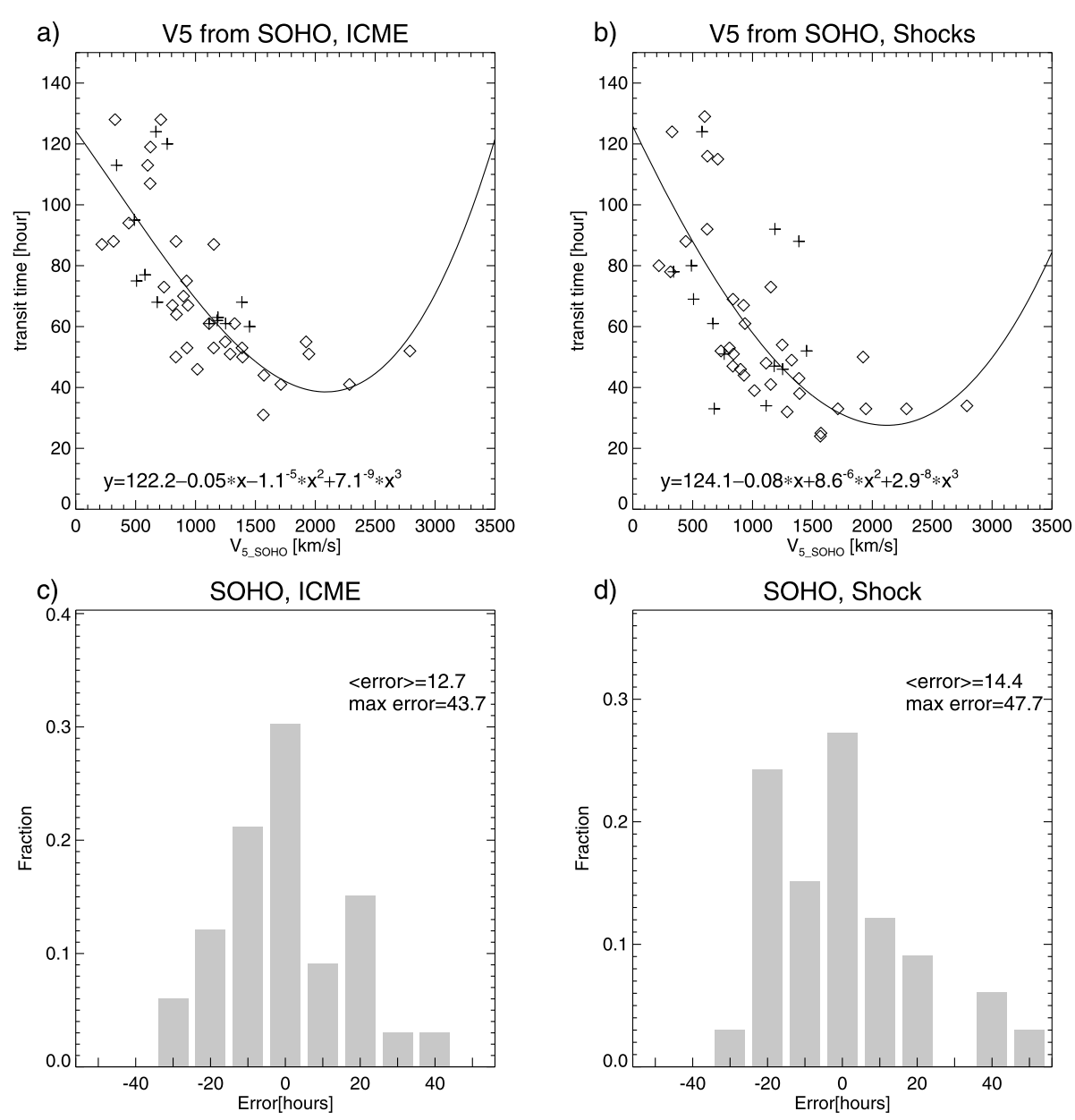

Figure 4 Relationship between the maximal/ $V_{5}$ CME speed and the observed TT for ICME (Panel a) and IP shock (Panel b). Open diamond symbols are for non-interacting and star symbols are for interacting CMEs, respectively. The third-order polynomial relationship between the observed TT and the speed is indicated by a dashed curve. The distributions of errors between predicted and observed TTs are presented in the bottom panels (Panel $\mathbf{c}$ for ICME and Panel $\mathbf{d}$ for IP shock). The values of the average and maximal errors are presented in the panels.

eration stops when the CME speed reaches that of the solar wind (i.e. $300-400 \mathrm{~km} \mathrm{~s}^{-1}$ ). From this moment onwards the CME moves with constant velocity, which is recorded at the Earth $\left[V_{\text {FINAL }}\right]$. Knowing the acceleration and using the above assumptions, we can easily calculate TT. The data we have allows us to determine the effective accelerations of CMEs (Gopalswamy et al. 2001b). They are designated as the quotient of the speed difference determined in the vicinity of the Sun and that determined at the Earth's vicinity over the TTs of CMEs. It can be expressed by the equation

$$
\mathrm{ACC}_{\text {EFFECTIVE }}=\left(V_{\text {INITIAL }}-V_{\text {FINAL }}\right) / \mathrm{TT},
$$


a) avg velocity from STEREO, ICME
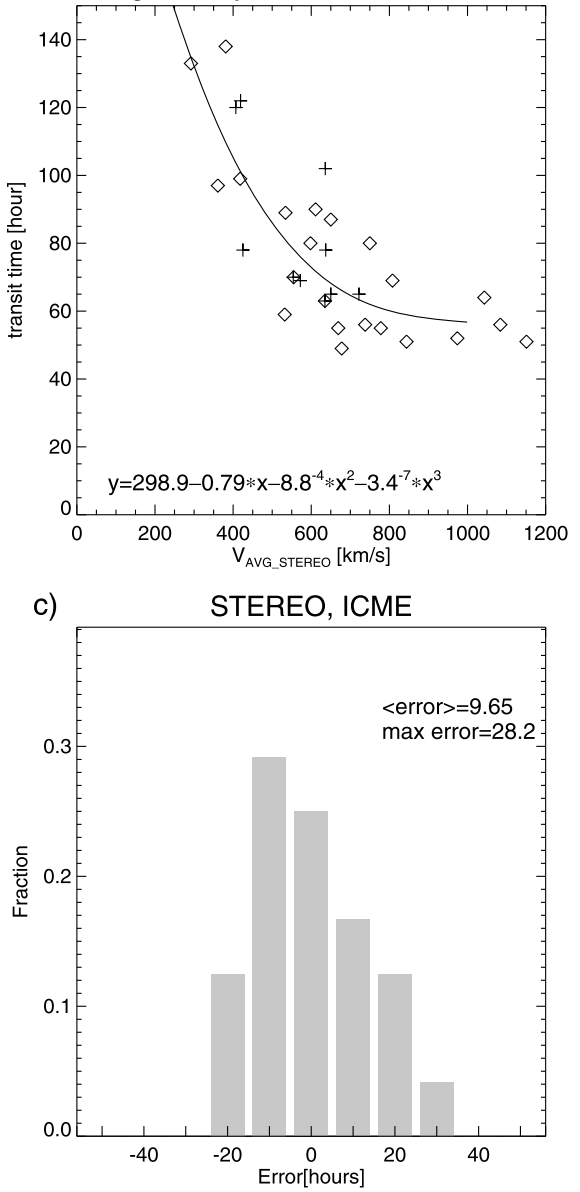

b) avr velocity from STEREO, Shock

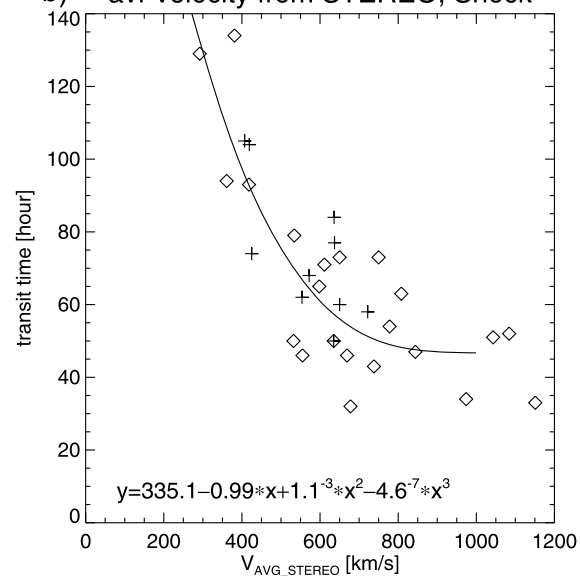

d) STEREO, Shock

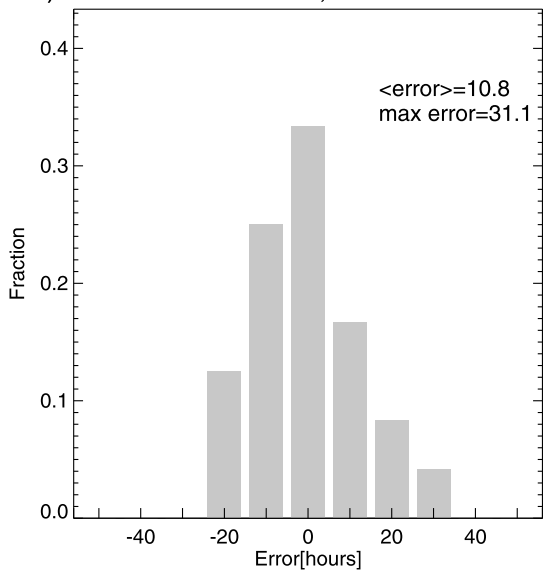

Figure 5 Relationship between the average CME speed obtained from the STEREO images and the observed TT for ICME (Panel a) and IP shock (Panel b). Open diamond symbols are for non-interacting and star symbols for interacting CMEs, respectively. The third-order polynomial relationship between the observed TT and the speed is indicated by a dashed curve. The distributions of errors between predicted and observed TT are presented in the bottom panels (Panel $\mathbf{c}$ for ICME and Panel $\mathbf{d}$ for IP shock). The values of average and maximal errors are presented in the panels.

where $V_{\text {INITIAL }}$ is velocity of CME determined near the Sun (average or maximal $/ V_{5}$ ) and $V_{\text {FINAL }}$ is velocity of ICME measured at the Earth's vicinity and TT, is the transit time of a given $\mathrm{CME}$ that is defined in the previous sections.

The relationship between initial velocities ( $V_{\mathrm{AVG}-\mathrm{SOHO}}, V_{5-\mathrm{SOHO}}, V_{\mathrm{AVG}-\mathrm{STEREO}}$, $V_{5 \text {-STEREO }}$ ) of CMEs and their effective accelerations are shown in Figure 7 . The quadratic relationship between the effective accelerations and the respective speeds are indicated by a solid line. These curves represent empirical models of the effective acceleration of CMEs depending on their initial speeds. Michałek et al. (2004) demonstrated that the average acceleration models cannot give an accurate prediction of TTs. They proposed to introduce the effective acceleration of a CME which is computed using a linear fit to two extreme samples of CMEs. The slowest events have no acceleration and the fastest events are accelerated up 

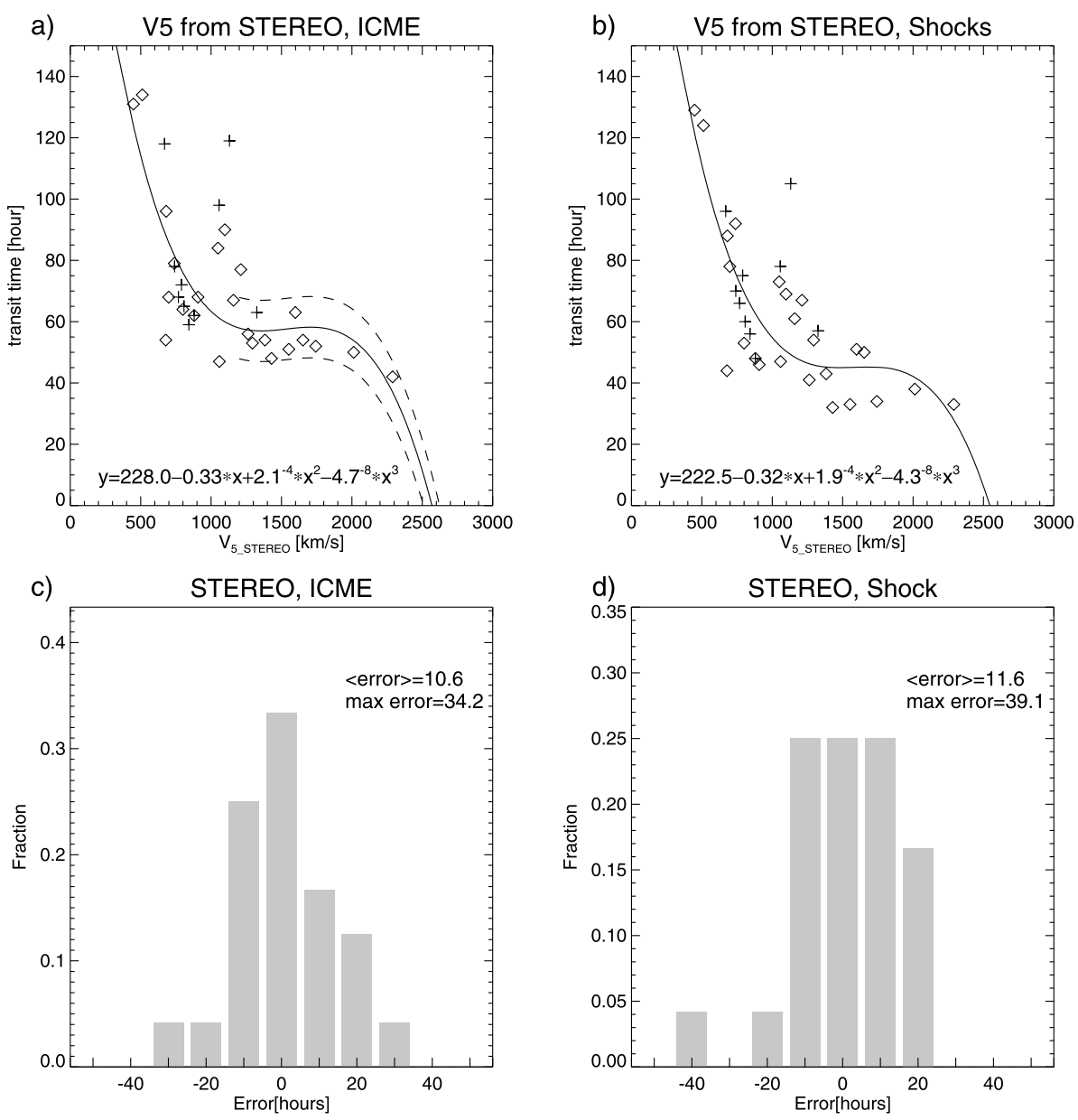

Figure 6 Relationship between the maximal $/ V_{5} \mathrm{CME}$ speed obtained from the STEREO images and the observed TT for ICME (Panel a) and IP shock (Panel b). Open diamond symbols are for non-interacting and star symbols are for interacting CMEs, respectively. The third-order polynomial relationship between the observed TT and the speed is indicated by a dashed curve. The distributions of errors between predicted and observed TTs are presented in the bottom panels (Panel $\mathbf{c}$ for ICME and Panel $\mathbf{d}$ for IP shock). The values of the average and maximal errors are presented in the panels. Two additional dashed lines illustrate deviation from the model of \pm 10 hours.

to $1 \mathrm{AU}$. These events are expected to have no acceleration cessation during their travel to the Earth (Michałek et al. 2004). The dashed lines in Figure 7 are linear fits to these groups of CMEs only.

With the help of different CME acceleration empirical models, we are able to determine the TT using the general equations of motion. The results are displayed in Figures 8 and 9. The scatter plots below display the observed versus predicted TT for the respective acceleration models displayed in Figure 7, which also shows a comparison of TTs for SOHO observations. Unfortunately, the results are not very accurate. Average errors for models obtained from the average and maximal speeds are above 13 and 15 hours, respectively. 

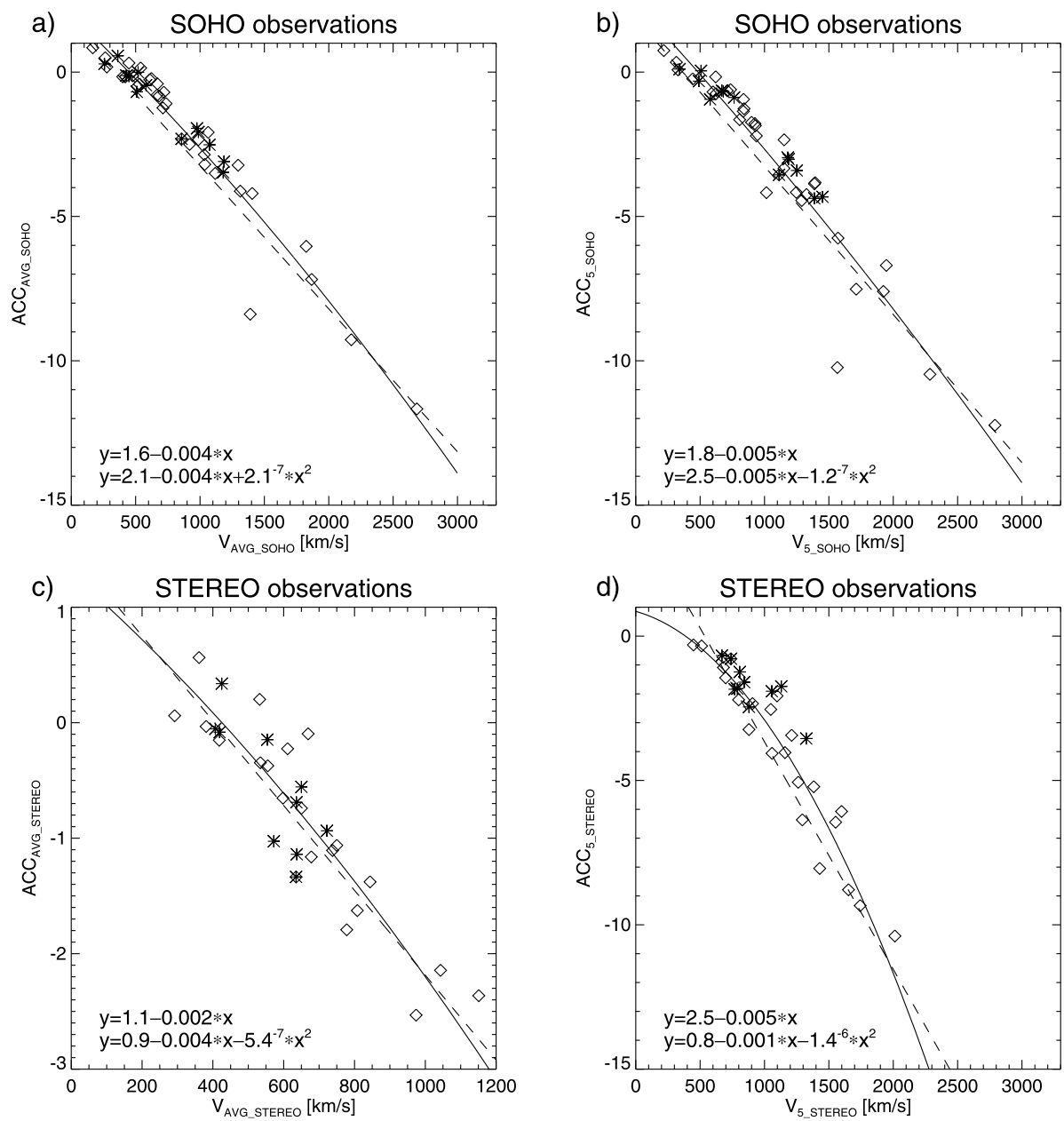

Figure 7 Scatter plots of effective acceleration versus respective initial speed of CMEs $\left[V_{\mathrm{AVG}}-\mathrm{SOHO}\right.$, $\left.V_{5-\mathrm{SOHO}}, V_{\mathrm{AVG}-\mathrm{STEREO}}, V_{5-\mathrm{STEREO}}\right]$. The open diamond symbols are for non-interacting and star symbols for interacting CMEs. The solid curves are quadratic fits to the data points. The dashed lines are linear fits for the three slowest and the three fastest events from the entire sample of CMEs.

As seen from the figure, the results seem to be better for effective accelerations determined from linear fits (Panels b and d) in comparison to those received from quadratic fits (Panels a and c). For the effective acceleration models obtained from the average velocities, data points are symmetrically scattered around the solid line, which shows the ideal situation when both times are equal. In the case of the maximal velocities the data points are mostly placed below this line. This means that on average the predicted TT are lower than observed TT.

In the figure, we have similar comparisons but for STEREO observations. In this case the results are more promising. For all of the models considered, the average errors are $\approx 9-12$ hours. However, the best results are for the effective acceleration obtained from the average velocities (Panels a and b). As seen from the figures, the average and maximal errors are 
a) avg $\mathrm{SOHO}$ speed, quadratic fit

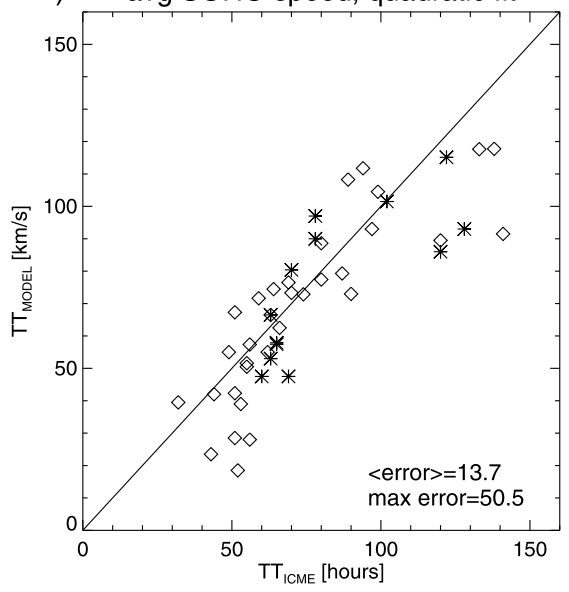

c) maximal $\mathrm{SOHO}$ speed, quadratic fit

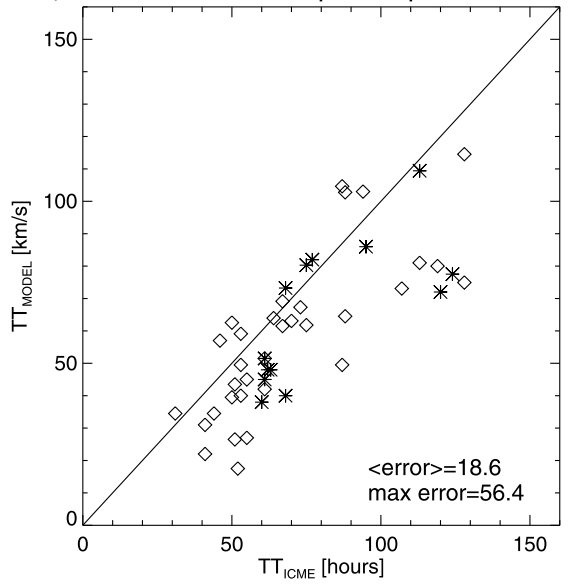

b) avg SOHO speed, linear fit

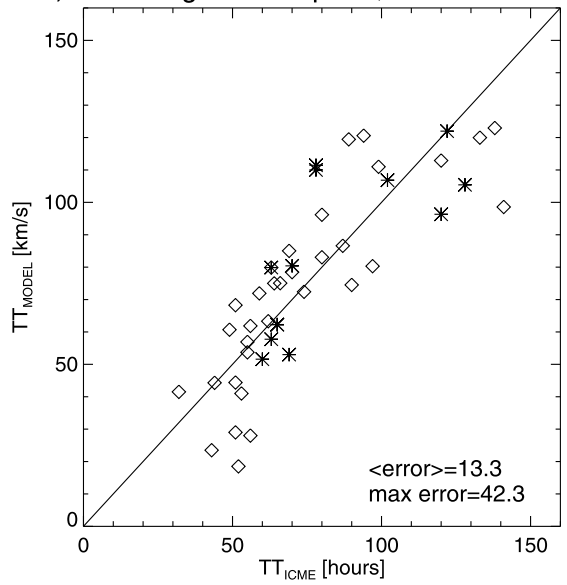

d) maximal $\mathrm{SOHO}$ speed, linear fit

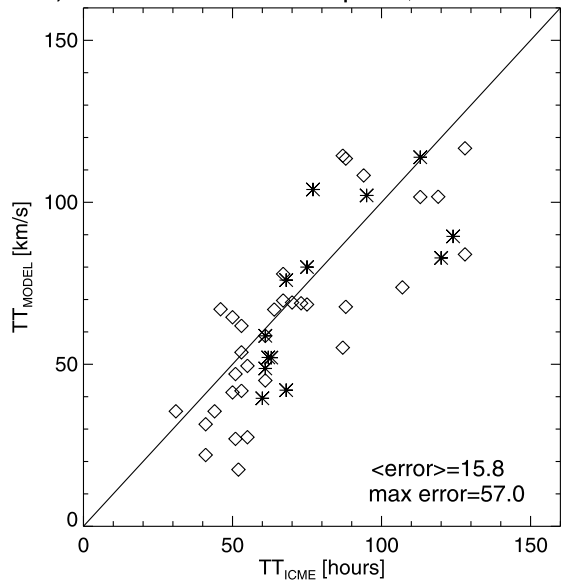

Figure 8 Scatter plots presenting comparison between observed and predicted TT based on acceleration profiles obtained from SOHO observations. In the respective panels we have results for effective acceleration obtained from a quadratic fit (Panels $\mathbf{a}$ and $\mathbf{b}$ ) and from a linear fit (Panels $\mathbf{c}$ and $\mathbf{d}$ ). The upper panels are for effective acceleration obtained from the average velocities and the bottom panels are from the maximal $/ V_{5}$ velocities, respectively. The open diamond symbols are for non-interacting and star symbols for interacting CMEs. Solid lines show the theoretical model when observed and predicted times are identical.

smallest and the symmetric scatter of the data points around the solid line represents the ideal situation when the observed and predicted TT are equal.

\subsection{Errors Versus the Range of CME Observation from the Sun}

The results obtained show that the best accuracy in predicting TT is provided by models based on the average speed determined from the STEREO observations (Figure 5a and Figure 9b). Using these two models the predicted TT are subject to minimal ( 9.27 hours, 10.2 hours) and maximal (28.3 hours, 26.6 hours) errors, respectively. 
a) avg STEREO speed, quadratic fit

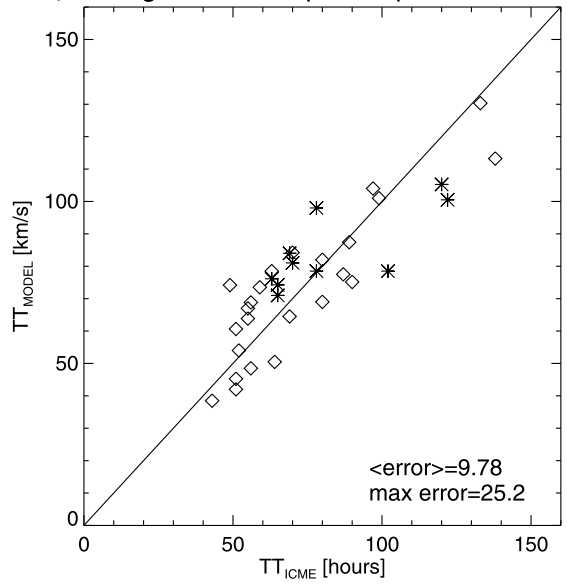

c) maximal STEREO speed, quadratic fit

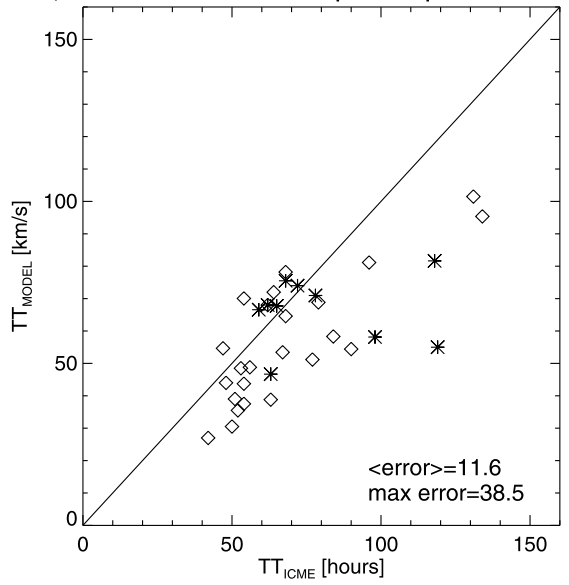

b) avg STEREO speed, linear fit

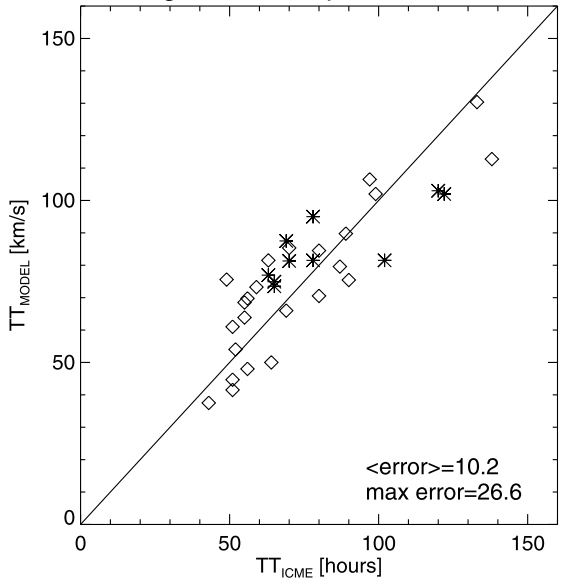

d) maximal STEREO speed, linear fit

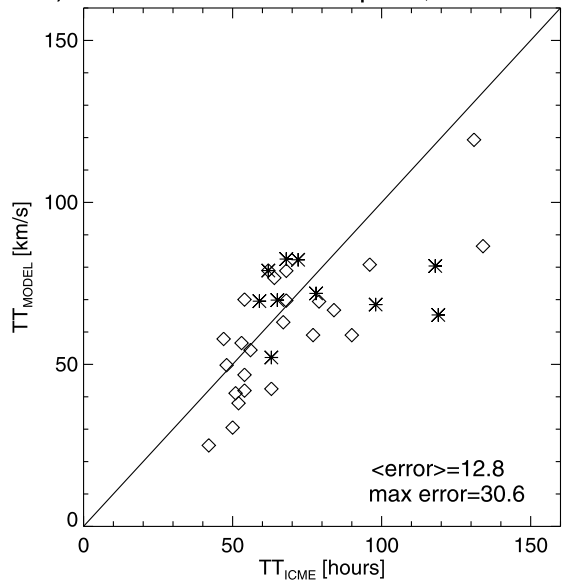

Figure 9 Scatter plots showing comparison between observed and predicted TT based on acceleration profiles obtained from the STEREO observations. In the respective panels we have results for effective acceleration obtained from a quadratic fit (Panels $\mathbf{a}$ and $\mathbf{b}$ ) and from a linear fit (Panels $\mathbf{c}$ and $\mathbf{d}$ ). The upper panels are for effective acceleration obtained from the average velocities and the bottom panels are from the maximal $/ V_{5}$ velocities, respectively. The open diamond symbols are for non-interacting and star symbols for interacting CMEs. Solid lines show the theoretical model when observed and predicted TT are identical.

It is important to consider why these observations provide the best results. Certainly, the direction of observation has some influence. It seems that observations from the STEREO spacecraft, in the case of a CME directed toward the Earth, are subject to smaller projection effects than those conducted with the SOHO spacecraft. However, another important factor to be considered is the field of view of the STEREO telescopes, which is much larger than the field of view of the SOHO coronagraphs. This effect is illustrated in Figure 10. This figure shows scatter plots of the difference between predicted (errors of predictions) and observed TT versus range of observations of CME $\left[D_{\mathrm{MAX}}\right]$. The parameter $\left[D_{\mathrm{MAX}}\right]$ is the maximal distance where a given CME was recorded by the STEREO telescopes. The left panel presents the results for the empirical model shown in Figure 5a and the right panel 

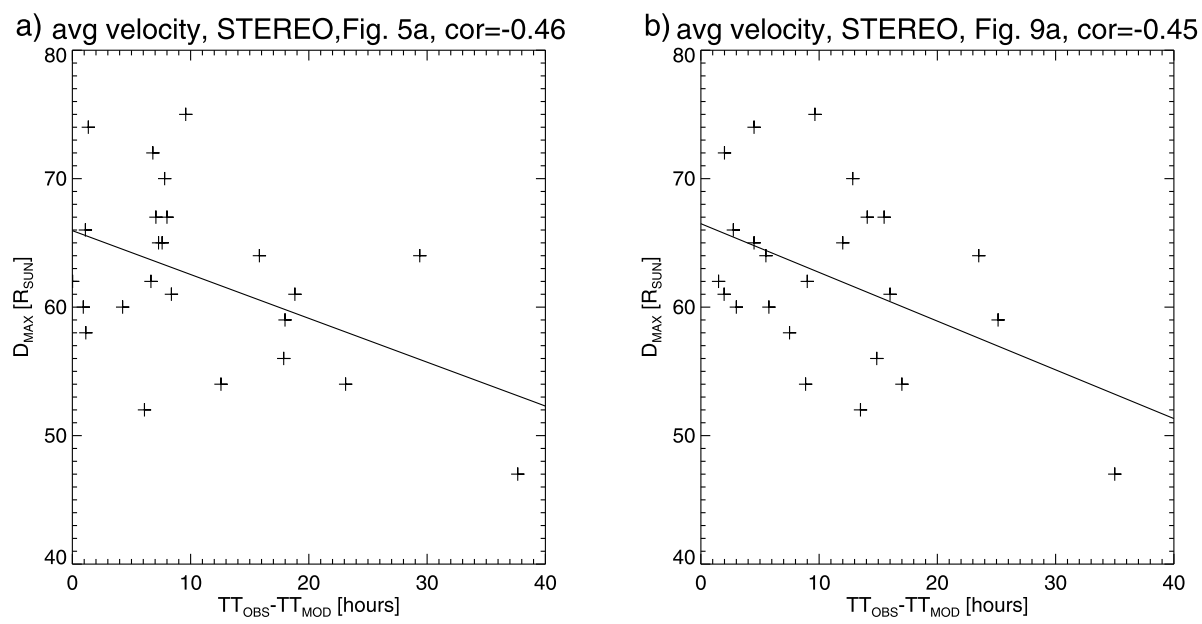

Figure 10 Scatter plots of difference between predicted (errors of predictions) and observed TT versus range of observations of CME [ $\left.D_{\mathrm{MAX}}\right]$. The left panel presents the results for the empirical model shown in Figure 5a and the right panel presents the results for calculations displayed in Figure 9a.

presents the results for calculation displayed in Figure 9a. As shown here, the errors in prediction depend on the range of observations. The correlation coefficients are $\approx 0.5$. This result is consistent with considerations presented by Bronarska and Michalek (2018). They showed that the errors in determining the speed depend, to the highest degree, on the number of height-time points. The average speeds determined from the STEREO observation have the highest number of height-time points and are therefore determined with the best accuracy.

\section{Summary and Discussion}

In this study we evaluate the TT of CMEs during the ascending phase of Solar Cycle 24. For this purpose we employed, different from the previous studies, two additional STEREO coronagraphs observing the Sun in quadrature and provided a new definition of the initial speed of CMEs. It was demonstrated that all of the considered initial speeds $\left[V_{\mathrm{AVG}-\mathrm{SOHO}}\right.$, $\left.V_{5-\text { SOHO }}, V_{\text {AVG-STEREO }}, V_{5 \text {-STEREO }}\right]$ are significantly correlated, however, the most significant correlation appears to be between the velocities determined using the same spacecrafts. The maximal velocities are larger by about $80 \%$ and $25 \%$ than the average velocities for the STEREO and SOHO telescopes, respectively. The initial velocities determined in the STEREO images $\left[V_{\mathrm{AVG}-\mathrm{STEREO}}, V_{5-\mathrm{STEREO}}\right]$ are also significantly related to the final velocities of an ICME. This is a very important result from the point of view of space weather. This allows us to accurately predict one of the most important parameters determining the geoeffectiveness of CMEs.

The TT of CMEs to the Earth is the next important parameter for space weather. We presented two methods, using the initial velocities of CMEs estimated near the Sun, to predict the TT. First, we used the correlation between the initial velocities and the TT to generate empirical models predicting the TT. The best empirical models are for the average speeds estimated in the STEREO images (Figure 4a). Second, we calculated the TT using kinematic 
equations of motions and different models of effective acceleration. Again the best results were obtained for average velocities determined in the STEREO field of view (Figure 9a).

Using observations from STEREO we were able to reduce the average absolute errors of TT prediction by only about an hour. However, what is more important is the fact that the new approach has radically reduced the maximum TT estimation errors to 29 hours. Previous studies determined the TT with the maximum error equal 50 hours. We also tried to find why the STEREO observations are more useful in determining the TT. As the STEREO field of view is much larger than the SOHO field of view, it allows us to follow the CME up to one third of the way to the Earth and thus to more accurately determine their speed. It is shown that the errors in predicting TT significantly depend on the distance range of the CME observation, i.e. the larger the observation range, the smaller the error.

In our research we defined a new initial velocity of a CME, the maximum velocity determined from the velocity profiles obtained from a moving linear fit to five consecutive height-time points. This new approach has radically reduced the maximum TT errors to 29 hours. Previous studies determined the TT with the maximum error equal 50 hours. Additionally, the maximal velocities of CMEs are better correlated with the final ICME speeds in comparison with previous models. It is also worth noting that in the case of maximum speeds, the empirical model obtained from the correlation between these speeds and TT are in good agreement. Results for these considerations are shown in Figure 6. For the fast CMEs $\left(V>1200 \mathrm{~km} \mathrm{~s}^{-1}\right)$, the empirical model works very well, as the average errors are below five hours and the maximal error does not exceed ten hours.

The model presented can be used universally. As input to this model, we can use speeds or accelerations obtained in different ways. In particular, we can use the real three dimensional speeds estimated using stereoscopic observations from all coronagraphs. It seems, however, that the results obtained in this way will not be significantly different from those obtained from the STEREO observations. As we mentioned earlier, STEREO observations in quadrature, in the case of halo CMEs, provide velocities very close to the real ones.

Acknowledgements Anitha Ravishankar and Grzegorz Michalek were supported by NCN through the grant UMO-2017/25/B/ST9/00536.

Disclosure of Potential Conflicts of Interest The authors declare that they have no conflicts of interest.

Publisher's Note Springer Nature remains neutral with regard to jurisdictional claims in published maps and institutional affiliations.

Open Access This article is distributed under the terms of the Creative Commons Attribution 4.0 International License (http://creativecommons.org/licenses/by/4.0/), which permits unrestricted use, distribution, and reproduction in any medium, provided you give appropriate credit to the original author(s) and the source, provide a link to the Creative Commons license, and indicate if changes were made.

\section{References}

Bronarska, K., Michalek, G.: 2018, Determination of projection effects of CMEs using quadrature observations with the two STEREO spacecraft. Adv. Space Res. 62, 408. DOI. ADS.

Brueckner, G.E., Howard, R.A., Koomen, M.J., Korendyke, C.M., Michels, D.J., Moses, J.D., Socker, D.G., Dere, K.P., Lamy, P.L., Llebaria, A., Bout, M.V., Schwenn, R., Simnett, G.M., Bedford, D.K., Eyles, C.J.: 1995, The Large Angle Spectroscopic Coronagraph (LASCO). Solar Phys. 162, 357. DOI. ADS.

Gopalswamy, N.: 2002, Relation between coronal mass ejections and their interplanetary counterparts. In: Wang, H., Xu, R. (eds.) Solar-Terrestrial Magnetic Activity Space Environ. 14, 157. ADS. 
Gopalswamy, N., Kaiser, M.L., Lepping, R.P., Kahler, S.W., Ogilvie, K., Berdichevsky, D., Kondo, T., Isobe, T., Akioka, M.: 1998, Origin of coronal and interplanetary shocks: a new look with WIND spacecraft data. J. Geophys. Res. 103, 307. DOI. ADS.

Gopalswamy, N., Lara, A., Lepping, R.P., Kaiser, M.L., Berdichevsky, D., St. Cyr, O.C.: 2000a, Interplanetary acceleration of coronal mass ejections. Geophys. Res. Lett. 27, 145. DOI. ADS.

Gopalswamy, N., Kaiser, M.L., Thompson, B.J., Burlaga, L.F., Szabo, A., Lara, A., Vourlidas, A., Yashiro, S., Bougeret, J.-L.: 2000b, Radio-rich solar eruptive events. Geophys. Res. Lett. 27, 1427. DOI. ADS.

Gopalswamy, N., Yashiro, S., Kaiser, M.L., Howard, R.A., Bougeret, J.-L.: 2001a, Characteristics of coronal mass ejections associated with long-wavelength type II radio bursts. J. Geophys. Res. 106, 29219. DOI. ADS.

Gopalswamy, N., Lara, A., Yashiro, S., Kaiser, M.L., Howard, R.A.: 2001b, Predicting the 1-AU arrival times of coronal mass ejections. J. Geophys. Res. 106, 29207. DOI. ADS.

Gopalswamy, N., Yashiro, S., Michałek, G., Kaiser, M.L., Howard, R.A., Reames, D.V., Leske, R., von Rosenvinge, T.: 2002, Interacting coronal mass ejections and solar energetic particles. Astrophys. J. 572, L103. DOI. ADS.

Gopalswamy, N., Yashiro, S., Michalek, G., Stenborg, G., Vourlidas, A., Freeland, S., Howard, R.: 2009, The SOHO/LASCO CME catalog. Earth Moon Planets 104, 295. DOI. ADS.

Kaiser, M.L., Kucera, T.A., Davila, J.M., St. Cyr, O.C., Guhathakurta, M., Christian, E.: 2008, The STEREO mission: an introduction. Space Sci. Rev. 136, 5. DOI. ADS.

Kim, R.-S., Cho, K.-S., Moon, Y.-J., Kim, Y.-H., Yi, Y., Dryer, M., Bong, S.-C., Park, Y.-D.: 2005, Forecast evaluation of the coronal mass ejection (CME) geoeffectiveness using halo CMEs from 1997 to 2003. J. Geophys. Res. 110, A11104. DOI. ADS.

Manoharan, P.K.: 2006, Evolution of coronal mass ejections in the inner heliosphere: a study using white-light and scintillation images. Solar Phys. 235, 345. DOI. ADS.

Manoharan, P.K.: 2010, Solar cycle changes of large-scale solar wind structure. In: Kosovichev, A.G., Andrei, A.H., Rozelot, J.-P. (eds.) Solar and Stellar Variability: Impact on Earth and Planets, IAU Symp. 264, Cambridge Univ. Press, Cambridge, 356. DOI. ADS.

Manoharan, P.K., Mujiber Rahman, A.: 2011, Coronal mass ejections - propagation time and associated internal energy. J. Atmos. Solar-Terr. Phys. 73, 671. DOI. ADS.

Manoharan, P.K., Gopalswamy, N., Yashiro, S., Lara, A., Michalek, G., Howard, R.A.: 2004, Influence of coronal mass ejection interaction on propagation of interplanetary shocks. J. Geophys. Res. 109, A06109. DOI. ADS.

Michałek, G., Gopalswamy, N., Yashiro, S.: 2003, A new method for estimating widths, velocities, and source location of halo coronal mass ejections. Astrophys. J. 584, 472. DOI. ADS.

Michalek, G., Gopalswamy, N., Yashiro, S.: 2017, CME velocity and acceleration error estimates using the bootstrap method. Solar Phys. 292, 114. DOI. ADS.

Michałek, G., Gopalswamy, N., Lara, A., Manoharan, P.K.: 2004, Arrival time of halo coronal mass ejections in the vicinity of the Earth. Astron. Astrophys. 423, 729. DOI. ADS.

Moon, Y., Cho, K., Dryer, M., Kim, Y., Bong, S., Chae, J., Park, Y.: 2005, New geoeffective parameters of very fast Halo Coronal Mass. In: AGU Spring Meeting Abstracts, SH23A. ADS.

Shanmugaraju, A., Vršnak, B.: 2014, Transit time of coronal mass ejections under different ambient solar wind conditions. Solar Phys. 289, 339. DOI. ADS.

Shanmugaraju, A., Syed Ibrahim, M., Moon, Y.-J., Mujiber Rahman, A., Umapathy, S.: 2015, Empirical relationship between CME parameters and geo-effectiveness of halo CMEs in the rising phase of Solar Cycle 24 (2011 - 2013). Solar Phys. 290, 1417. DOI. ADS.

Smart, D.F., Shea, M.A.: 1985, A simplified model for timing the arrival of solar flare-initiated shocks. $J$. Geophys. Res. 90, 183. DOI. ADS.

Smith, Z., Dryer, M.: 1990, Magnetohydrodynamic study of temporal and spatial evolution of simulated interplanetary shocks in the ecliptic plane within 1-A.U. Solar Phys. 129, 387. DOI. ADS.

Srivastava, N., Venkatakrishnan, P.: 2002, Relationship between CME speed and geomagnetic storm intensity. Geophys. Res. Lett. 29, 1287. DOI. ADS.

Syed Ibrahim, M., Manoharan, P.K., Shanmugaraju, A.: 2017, Propagation of coronal mass ejections observed during the rising phase of Solar Cycle 24. Solar Phys. 292, 133. DOI. ADS.

Vršnak, B., Žic, T., Vrbanec, D., Temmer, M., Rollett, T., Möstl, C., Veronig, A., Čalogović, J., Dumbović, M., Lulić, S., Moon, Y.-J., Shanmugaraju, A.: 2013, Propagation of interplanetary coronal mass ejections: the drag-based model. Solar Phys. 285, 295. DOI. ADS.

Yashiro, S., Gopalswamy, N., Michalek, G., St. Cyr, O.C., Plunkett, S.P., Rich, N.B., Howard, R.A.: 2004, A catalog of white light coronal mass ejections observed by the SOHO spacecraft. J. Geophys. Res. 109, A07105. DOI. ADS.

Yashiro, S., Gopalswamy, N., Akiyama, S., Makela, P.A., Xie, H.: 2014, Association rate of major SEP events as a function of CME speed and source longitude. In: AGU Fall Meeting Abstracts 2014, SH43A. ADS. 